\section{Learning the histone language}

Sequential rounds of immunoprecipitation and barcoding reveal the genomewide occurrence of paired histone marks.

The genome is regulated by a complex interplay of DNA sequence and epigenetic modifications on DNA and histones. Much like in a language, meaning is derived from different combinations of these marks. Certain combinations activate genes, others keep genes in a poised state or repress their activity. Current methods to sequence chromatin look at one modification at a time, and although the data can be combined for analysis, it is not easy to reconstruct whether the marks occurred on the same molecule or even in the same cell.

Ido Amit from the Weizmann Institute tackles this question by profiling the cooccurrence of histone marks. During coChIP (combinatorial indexed chromatin immunoprecipitation), fragmented nucleosomes are first captured in wells with immobilized antibodies, each against a specific modification. The free DNA ends are then barcoded to index the mark, and all the different reactions are pooled. After a second capture step against a specific modification, another barcode is added, and sequencing of the barcode pairs allows one to reconstruct which marks occurred together.

The team looked at 70 different pairs of marks. But the method is not limited to pairs: "I don't see any technical reason why three or even four co-ChIP could not be performed," says Amit, but he adds that each round needs another immunoprecipitation step and hence more starting material.

Co-ChIP data reveal not only how the marks are read but also how they were written. From the pairs one can determine whether the marks were deposited or removed independently or interdependently, or whether some are mutually exclusive.

The profiling of bivalent domains - that is, the co-occurrence of an activating and a repressive mark-showed their emergence on developmental genes in embryonic stem cells (ESCs) that are primed for differentiation, whereas this combination of marks is still largely absent in naive ESCs. Many of these bivalent marks are lost once cells are fully differentiated, but the researchers saw de novo establishment in certain tissues. They concluded that bivalency may be a common regulatory mechanism that is employed beyond ESCs.

Co-ChIP's Achilles heel is its dependence on high-quality antibodies for immunoprecipitation. "We really have only a handful of such antibodies currently," says Amit.

Nicole Rusk

\section{RESEARCH PAPERS}

Weiner, A. et al. Co-ChIP enables genome-wide mapping of histone mark co-occurrence at singlemolecule resolution. Nat. Biotechnol. 34, 953-961 (2016). 